\title{
Gas Expansion Energy Model and Numerical Simulation of Outburst Coal Seam under Multifield Coupling
}

\author{
Jie Cao, ${ }^{1,2,3}$ Qianting Hu, ${ }^{1}$ Yanan Gao, ${ }^{4}$ Minghui Li $\mathbb{D}^{4,5}$ and Dongling Sun ${ }^{2,3}$ \\ ${ }^{1}$ School of Resources and Safety Engineering, Chongqing University, Chongqing 400044, China \\ ${ }^{2}$ State Key Laboratory of the Gas Disaster Detecting, Preventing and Emergency Controlling, Chongqing 400037, China \\ ${ }^{3}$ China Coal Technology and Engineering Group Chongqing Research Institute, Chongqing 400037, China \\ ${ }^{4}$ State Key Laboratory for GeoMechanics and Deep Underground Engineering, China University of Mining and Technology, \\ Xuzhou, China \\ ${ }^{5}$ Guangdong Provincial Key Laboratory of Deep Earth Sciences and Geothermal Energy Exploitation and Utilization, Institute of \\ Deep Earth Sciences and Green Energy, College of Civil and Transportation Engineering, Shenzhen University, \\ Shenzhen 518060, China
}

Correspondence should be addressed to Minghui Li; mhli@szu.edu.cn

Received 3 March 2021; Revised 2 April 2021; Accepted 9 April 2021; Published 21 April 2021

Academic Editor: Yang Wang

Copyright (C) 2021 Jie Cao et al. This is an open access article distributed under the Creative Commons Attribution License, which permits unrestricted use, distribution, and reproduction in any medium, provided the original work is properly cited.

Due to the insufficient understanding of the outburst mechanism, the coal and gas outburst disasters in China are more serious. Gas expansion energy is the main source of energy that causes outburst. In order to explore the distribution law of gas expansion energy in outburst coal seams, a gas-solid coupling equation of outburst coal seams was established. The distribution law of coal stress field, deformation field, gas flow field, and gas expansion energy were simulated and analyzed by using COMSOL Multiphysics. The results showed that from the excavation face to the deep part of coal seam, the stress presented unloading zone, stress concentration zone, and original stress zone. The volumetric strain and permeability reached the minimum, while the gas pressure reached the maximum at the peak value of vertical stress. As time goes on, the gas pressure in the fracture near the working face gradually decreased and was less than the pressure in coal matrix. The total gas expansion energy consists of free gas and desorption gas expansion energy. Affected by the excavation, free gas expansion energy maintained a constant value in the original coal seam and gradually decreased in the area close to the working face. The expansion energy provided by desorption gas was zero in the original coal seam. And it first increased and then decreased rapidly near the working face. Compared with stress and coal seam thickness, gas pressure and initial diffusion coefficient had significant influence on gas expansion energy of coal seam. When the diffusion coefficient was greater than $1 \mathrm{e}-9 \mathrm{~m}^{2} / \mathrm{s}$, the gas expansion energy of the coal seam near the working face was significantly higher than that of the original coal seam, which had the risk of inducing outburst.

\section{Introduction}

Coal and gas outburst is one of the most serious disasters that endanger coal mine safety production. In the past ten years, there have been 125 major coal and gas outburst accidents in China (more than or equal to 3 casualties in a single accident), with a total of 891 casualties. With the deepening of coal mining, the occurrence conditions of coal and gas are more complex, and the outburst accident shows new characteristics, which brings new challenges to the disaster early warning and prediction. From the perspective of energy anal- ysis, the occurrence of coal and gas outburst is due to the accumulation of energy in the coal and rock mass in front of the mining work to a certain extent during the mining operations, which destroys the original equilibrium state and release energy rapidly.

The former Soviet Union scholar Ходот [1] first proposed the energy theory of outburst. He believed that coal and gas outburst is caused by the deformation potential energy of coal and the gas expansion energy, and there is an outburst risk when the energy conditions are met. Zheng [2] analyzed the energy sources of particularly serious coal 
and gas outburst using the dimensional analysis method. Taking the outburst coal mass as the object, the elastic energy and gas expansion energy of coal were calculated, respectively. It was considered that the main energy of outburst comes from gas expansion energy. Wen [3] obtained a calculation model of gas internal energy and coal crushing work through a large number of tests and used the model to approximate actual outburst accident in Guizhou province in China. Lan [4] analyzed the outburst energy distribution under different initial gas pressures based on the basic algorithm of coal and rock elastic energy and gas expansion energy and believed that the degree of gas participation determines the relationship between the two energies. When the gas pressure is lower than $0.2 \mathrm{MPa}$, the elastic energy is the main energy; when the gas pressure is between 0.4 and $0.6 \mathrm{MPa}$, the two energies are basically the same in order of magnitude; when the gas pressure is greater than $0.6 \mathrm{MPa}$, the gas expansion energy far exceeds the elastic energy of coal and rock.

$\mathrm{Hu}$ et al. [5] and Wang et al. [6] established the energy relationship between coal and gas outburst under actual working conditions and obtained that when the roadway is excavated, the stress redistribution will be caused. The original elastic zone will be converted into plastic zone; the coal elastic energy and gas internal energy in this zone are released to induce outburst. It was believed that the gas content can reflect the gas expansion energy. A large number of studies showed that gas is the main energy source of outburst, and the energy provided by gas in the process of outburst is $1 \sim 3$ orders of magnitude of the energy provided by stress [7-11].

In recent years, in order to further explore the conditions of outburst energy, Jiang et al. $[12,13]$ proposed a critical gas release energy indicator. Through outburst simulation experiments, $42.98 \mathrm{~mJ} / \mathrm{g}$ was the critical value for outburst of coal seams $(103.8 \mathrm{~mJ} / \mathrm{g}$ was the critical value for strong outburst), and its accuracy had been verified on site. Afterwards, scholars carried out a series of studies on gas expansion energy. Gas expansion energy is directly related to the amount of gas involved in outburst work. The desorption experiments under different gas pressure, coal quality (gas-bearing tectonically deformed coal and primaryundeformed coal), and water content were carried out by Wang et al. [14, 15] using a self-developed desorption device. The results showed that the higher the gas pressure is, the greater the total gas expansion energy (TGEE) of coal is; the gas desorption rate of tectonically deformed coal is higher, and the TGEE of tectonically deformed coal is about 3 times of that of primary-undeformed coal; the TGEE of coal decreases with the increase of water content. Yang et al. [16] selected seven coal samples with different metamorphic degrees for outburst simulation experiment and obtained that the gas release amount of outburst coal samples was greater than that of nonoutburst coal samples in the first $10 \mathrm{~s}$. There was a good linear relationship between the initial gas release amount (the first 10 seconds) and the initial gas release expansion energy, which can reflect the risk of coal outburst. Through experimental research, Liang et al. [17] obtained that the influence of gas pressure, coal particle size, and water content on initial gas release expansion energy decreases in turn, and the gas expansion energy increases significantly with the increase of gas pressure. Xue et al. [18] obtained that the minimum energy required for outburst is about $1.1 \mathrm{MJ} / \mathrm{m}^{3}$; compared with the free gas energy, the desorption gas energy of coal plays a decisive role in the initiation and spread of outburst. Lu et al. [19] analyzed the outburst energy under three different conditions (normal coal, deformed coal, and coal combination) by constructing a numerical analysis model. Among the outburst energy of coal combination, the expansion energy of desorption gas is 1 6 times of elastic energy and 5 17 times of free gas energy. Zhao et al. [20] considered that rapid desorption in a short time is a necessary condition for the occurrence of outburst. At the same time, in the process of outburst development, the transportation of outburst coal needs a large amount of desorption gas. For Zhongliangshan outburst, the expansion energy of desorption gas for coal transportation was about $5.61 \times 10^{8} \mathrm{~J}$, which is nearly 6.3 times of that of free gas.

Researches on the gas expansion energy of outburst are mostly based on outburst simulation experiments. Due to the complexity and the diversity of influencing factors, the simulation experiment is often difficult and the amount of data is relatively limited. On the basis of experimental research, it is a reliable research method to analyze the evolution law of stress and gas pressure field in outburst coal seam by constructing multiphysical field coupling model and using numerical simulation software [21-24], which can make up for the lack of physical simulation experiment. Therefore, this paper intends to establish a gas expansion energy model considering the coupling of coal seam stress and gas desorption and seepage and uses COMSOL numerical simulation software to solve it. The influence of different factors on the gas expansion energy in front of the working face can be obtained, which can provide support for further establishment of quantitative relationship model of outburst occurrence and determination of disaster monitoring and early warning index.

\section{Governing Equations}

Coal and gas outburst is a complex mechanical process [6]. In the accumulation stage of outburst, the mining operation destroys the original stress balance, resulting in stress concentration, strain softening, deformation, or strength failure. Coal deformation leads to the change of porosity and permeability, which in turn changes the gas pressure in pores and fractures. On the one hand, the change of gas pressure in pore and fracture changes the effective stress of coal seam and induces deformation; on the other hand, it causes desorption and diffusion of adsorbed gas in coal matrix, which further causes shrinkage deformation of coal matrix and changes of flow field. Therefore, the gas-solid coupling equation of outburst coal seam consists of the deformation governing equation, the gas diffusion governing equation in coal matrix, and the gas seepage governing equation in coal fracture. Due to the complexity of the 
mechanism, the basic hypothesis and simplification were put forward in the analysis process:

(1) Coal seam is isotropic elastic-plastic porous medium

(2) The temperature factor in the process of coal deformation and gas desorption seepage is not considered

(3) The coal deformation behind the outburst hole wall is small

(4) Gas seepage obeys the generalized power law

2.1. Deformation Governing Equation. The deformation characteristics of outburst coal seam are determined by effective stress. The coal stress balance equation considering effective stress can be expressed as

$$
\begin{aligned}
& \sigma_{i j, j}-\left(\beta_{f} p_{f}+\beta_{m} p_{m}\right) \delta_{i j}+f_{i}=0, \\
& \beta_{m}=1-\frac{K}{K_{m}}, \beta_{f}=1-\frac{K}{K_{f}}, \\
& K=\frac{E}{3(1-2 v)}, K_{m}=\frac{E_{m}}{3(1-2 v)},
\end{aligned}
$$

where $\sigma_{i j}$ is the component of the total stress tensor, $\mathrm{MPa}$; $p_{f}$ and $p_{m}$ are the gas pressure in the coal fracture and the coal matrix, respectively, $\mathrm{MPa} ; \beta_{f}$ and $\beta_{m}$ are the Biot's coefficient; $\delta_{i j}$ is the Kronecker delta $\left(\delta_{i j}=1\right.$ as $i=j$ and 0 in other cases); $f_{i}$ is the body force of coal, MPa; $K$, $K_{m}$, and $K_{f}$ are the bulk modulus of coal, coal matrix particles and coal fracture, respectively, $\mathrm{MPa} ; E$ and $E_{m}$ are the elastic modulus of coal and coal matrix, $\mathrm{MPa}$; and $v$ is Poisson's ratio.

The strain and displacement of coal seam should satisfy the geometric equation, expressed by the following formula:

$$
\varepsilon_{i j}=\frac{1}{2}\left(u_{i, j}+u_{j, i}\right)
$$

where $\varepsilon_{i j}$ is the strain tensor of coal and $u_{i}(i=x, y, z)$ is the displacement of coal in the direction of $i$.

Therefore, the constitutive equation of the isotropic elastic body is

$$
\begin{gathered}
\sigma_{i j}=2 G \varepsilon_{i j}+\lambda \varepsilon_{v} \delta_{i j^{-}}\left(\beta_{f} p_{f}+\beta_{m} p_{m}\right) \delta_{i j}-K \varepsilon_{s} \delta_{i j}, \\
G=\frac{E}{2(1+v)}, \lambda=\frac{2 v}{1-2 v} G, \varepsilon_{s}=\frac{\varepsilon_{L} p_{m}}{p_{m}+P_{L}},
\end{gathered}
$$

where $G$ is the shear modulus of coal, MPa; $\lambda$ is the Lamé constant; $\varepsilon_{v}$ is the volumetric strain, $\varepsilon_{v}=\varepsilon_{11}+\varepsilon_{22}+\varepsilon_{33} ; \varepsilon_{s}$ is the coal adsorption swelling or desorption shrinkage strain; and $\varepsilon_{L}$ is Langmuir volumetric strain. Then, the equilibrium differential equation expressed by displacement is obtained as follows:

$$
G u_{i, j j}+\frac{G}{1-2 v} u_{j, j i}-\left(\beta_{f} p_{f, i}+\beta_{m} p_{m, i}\right) \delta_{i j}-K \varepsilon_{s} \delta_{i j}+f_{i}=0 .
$$

In this study, Drucker-Prager matching Mohr-Coulomb criterion is used to characterize the plastic instability of coal

$$
\left\{\begin{array}{l}
F=\frac{2 \sin \varphi}{\sqrt{3}(3-\sin \varphi)} I_{1}-\frac{6 c \cos \varphi}{\sqrt{3}(3-\sin \varphi)}+\sqrt{J_{2}} \\
I_{1}=\sigma_{1}+\sigma_{2}+\sigma_{3} \\
I_{2}=\sigma_{1} \sigma_{2}+\sigma_{2} \sigma_{3}+\sigma_{3} \sigma_{1} \\
J_{2}=\frac{1}{3} I_{1}^{2}-I_{2}
\end{array},\right.
$$

where $I_{1}$ represents the first stress invariant; $J_{2}$ represents the second deviator stress invariant; $c$ is the cohesion, MPa; $\varphi$ is the friction angle, .

2.2. The Governing Equation of Gas Diffusion in Coal Matrix. The mass conservation equation of gas in the coal matrix is

$$
\frac{\partial m}{\partial t}=-Q_{s}
$$

where $t$ is the time, $s ; m$ is the gas mass per unit volume of coal, composed of adsorbed gas and free gas, $\mathrm{kg} / \mathrm{m}^{3}$, which can be calculated by Langmuir equilibrium equation and ideal gas state equation.

$$
m=\frac{M_{c} \rho_{g}}{V_{M}} \frac{V_{L} p_{m}}{p_{m}+P_{L}}+\phi_{m} \frac{M_{c}}{R T} p_{m}
$$

where $M_{c}$ is the molar mass of methane, $\mathrm{kg} / \mathrm{mol} ; \rho_{g}$ is the gas density under standard state, $\mathrm{kg} / \mathrm{m}^{3} ; V_{M}$ is the molar volume of methane under standard conditions, $\mathrm{m}^{3} / \mathrm{mol} ; V_{L}$ is the maximum adsorption capacity of coal, $\mathrm{mL} / \mathrm{g} ; P_{L}$ is the Langmuir pressure constant of coal, $\mathrm{Pa} ; \phi_{m}$ is the porosity in coal matrix, \%; $R$ is the universal gas constant, $\mathrm{J} /(\mathrm{mol} \cdot \mathrm{K})$; and $T$ is the temperature, K. Gas diffusion in coal matrix and fractures is caused by concentration gradient, which is considered as unsteady diffusion and can be expressed by the following equation [25]:

$$
Q_{s}=\frac{3 \pi^{2} M_{c}\left(p_{m}-p_{f}\right) D_{0} \exp (-\lambda t)}{L_{m}^{2} R T},
$$

where $D_{0}$ is the initial diffusion coefficient, $\mathrm{m}^{2} / \mathrm{s} ; \lambda$ is the correlation coefficient; and $L_{m}$ is the width of coal matrix, m. 


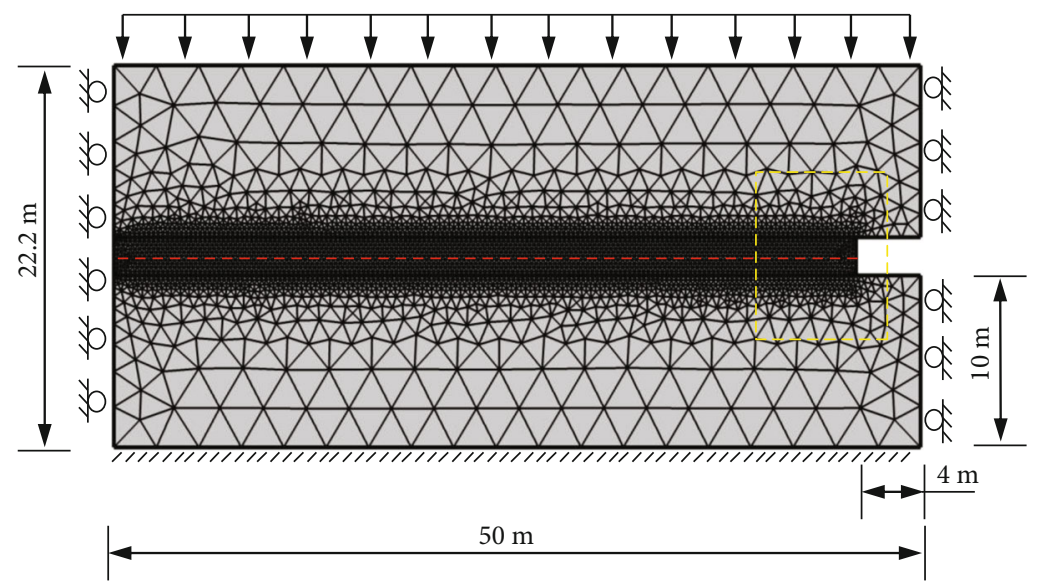

FIgURE 1: Geometric model.

Substituting the equations (7) and (8) into the governing equation (6), we can get

$$
\frac{\partial p_{m}}{\partial t}=-\frac{3 \pi^{2}\left(p_{m}-p_{f}\right) D_{0} \exp (-\lambda t) V_{M}\left(p_{m}+P_{L}\right)^{2}}{V_{L} \rho_{g} P_{L} R T+\phi_{m} V_{M}\left(p_{m}+P_{L}\right)^{2}} .
$$

2.3. The Governing Equation of Gas Seepage in Coal Fracture. The movement of gas in coal fracture accords with the continuity equation

$$
\begin{aligned}
\frac{\partial}{\partial t}\left(\phi_{f} \rho_{f}\right)+\nabla \cdot\left(\rho_{f} V_{f}\right) & =Q_{s}\left(1-\phi_{f}\right), \\
\rho_{f} & =\frac{M_{c} p_{f}}{R T}
\end{aligned}
$$

where $\phi_{f}$ is the porosity in the coal fracture, \%; and $V_{f}$ is the gas seepage rate in the fracture. Due to the discreteness of fracture development, the gas flow patterns are different, which can be determined by the local Reynolds number, and its movement can be described by the generalized power law.

$$
V_{f}=-\frac{k_{f}}{\mu}\left(\nabla p_{f}\right)^{m}
$$

where $k_{f}$ is the permeability, $\mathrm{m}^{2} ; \mu$ is gas dynamic viscosity, $\mathrm{Pa}$ - $s$; and $m$ is gas state index, $m=1 \sim 2$, when $m=1$, the above formula is Darcy seepage model. Disturbance of coal seam stress causes the porosity and permeability changes. The porosity equation in fracture is [26]

$\frac{\phi_{f}}{\phi_{f 0}}=1-\frac{K_{m}}{K_{f} L_{m}+K_{m} L_{f}}\left[\frac{P_{L} L_{m} \varepsilon_{L}\left(p_{m}-p_{m 0}\right)}{\left(p_{m}+P_{L}\right)\left(p_{m 0}+P_{L}\right)}-\left(L_{m}+L_{f}\right) \varepsilon_{V}\right]$,

where $L_{f}$ is the width of coal fractures, m. According to the cubic law [27], the relationship between permeability and porosity can be obtained

$$
\frac{k_{f}}{k_{f 0}}=\left(\frac{\phi_{f}}{\phi_{f 0}}\right)^{3}
$$

TABLE 1: The physical parameters.

\begin{tabular}{lc}
\hline Parameters and units & Value \\
\hline Elasticity modulus of coal $E /(\mathrm{GPa})$ & 3 \\
Elasticity modulus of coal matrix $E_{m} /(\mathrm{GPa})$ & 8 \\
Poisson's ratio of coal $v$ & 0.32 \\
Density of coal $\rho_{c} /\left(\mathrm{kg} / \mathrm{m}^{3}\right)$ & 1350 \\
Elasticity modulus of coal $E r /(\mathrm{GPa})$ & 30 \\
Poisson's ratio of rock $v_{r}$ & 0.2 \\
Density of rock $\rho_{r} /\left(\mathrm{kg} / \mathrm{m}^{3}\right)$ & 2450 \\
Cohesion of coal $c /(\mathrm{MPa})$ & 1.45 \\
Friction angle of coal $\varphi /\left(^{\circ}\right)$ & 30 \\
Langmuir pressure constant of coal $P_{L} /(\mathrm{MPa})$ & 1 \\
Langmuir constant volume of coal $V L\left(\mathrm{~m}^{3} / \mathrm{kg}\right)$ & 0.02 \\
Initial matrix porosity of coal $\varphi_{m 0}$ & 0.03 \\
Initial fracture porosity of coal $\varphi_{f 0}$ & 0.001 \\
Initial permeability $k_{f 0}\left(\mathrm{~m}^{2}\right)$ & $1 \mathrm{e}-13$ \\
Gas dynamic viscosity $\mu /(\mathrm{Pa} \cdot \mathrm{s})$ & $1.52 \mathrm{e}-5$ \\
Initial diffusion coefficient $D_{0} /\left(\mathrm{m}^{2} / \mathrm{s}\right)$ & $5 \mathrm{e}-11$ \\
\hline
\end{tabular}

By substituting (8), (12) into (10), the governing equation of gas seepage in fracture is obtained as follows:

$$
\begin{aligned}
\phi_{f} \frac{\partial \rho_{f}}{\partial t}+\rho_{f} \frac{\partial \phi_{f}}{\partial t}-\nabla \cdot\left(\frac{k_{f}}{\mu} \rho_{f}\left(\nabla p_{f}\right)^{m}\right) \\
=\frac{3 \pi^{2} M_{c} D_{0} \exp (-\lambda t)\left(1-\phi_{f}\right)}{L_{m}^{2} R T}\left(p_{m}-p_{f}\right) .
\end{aligned}
$$

Simultaneous formulas (4), (5), (9), (12) (15) are the mathematical model of multiphysical fields of coal and gas outburst seam.

\section{Gas Expansion Energy Calculation and Numerical Simulation}

3.1. Gas Expansion Energy Calculation. The gas expansion energy $W$ in the process of outburst is composed of the free 


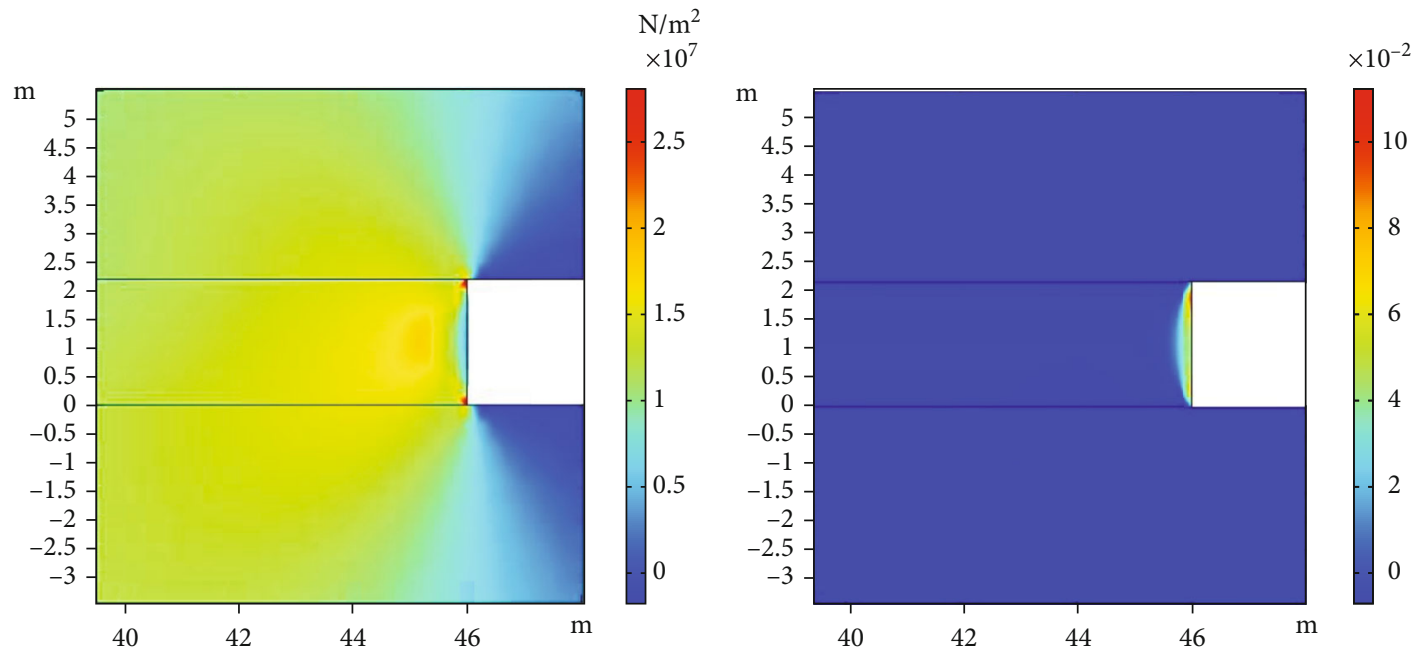

(a) Vertical stress, $\mathrm{Pa}$

(b) Volumetric strain

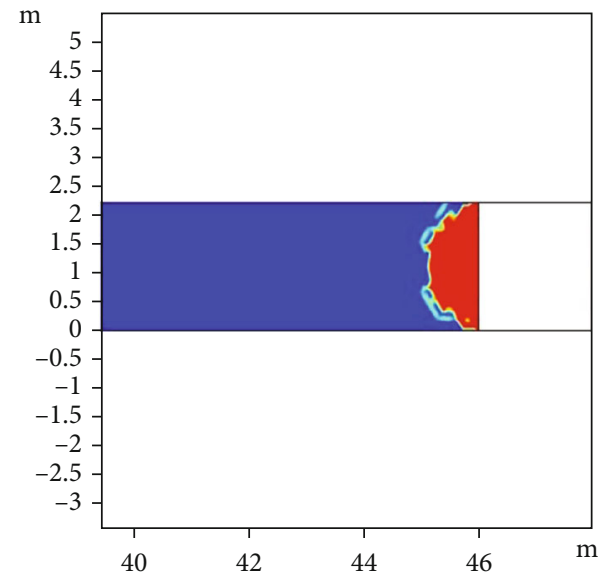

(c) Plastic strain

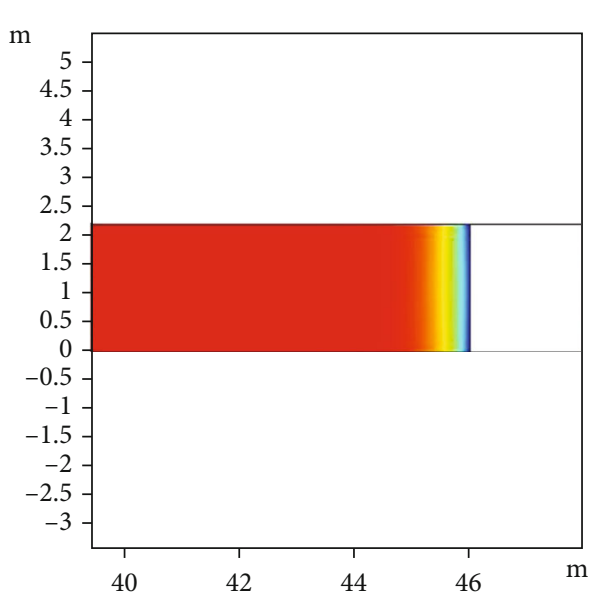

(e) Gas pressure in matrix, $\mathrm{Pa}$
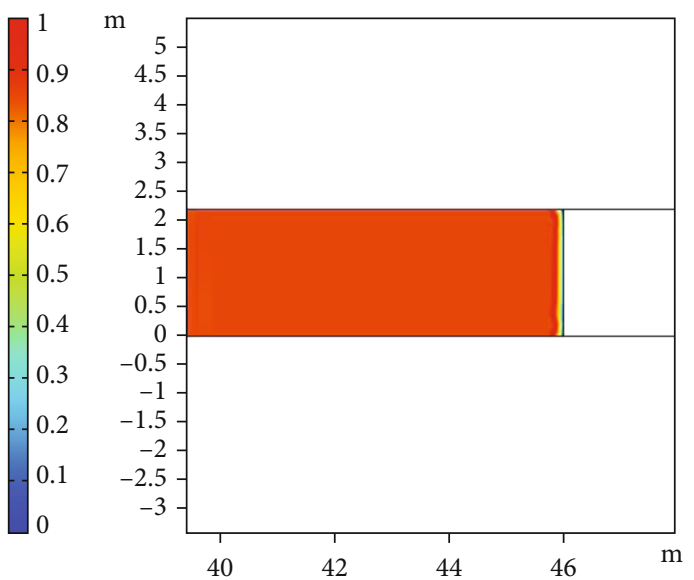

(d) Gas pressure in fracture, $\mathrm{Pa}$
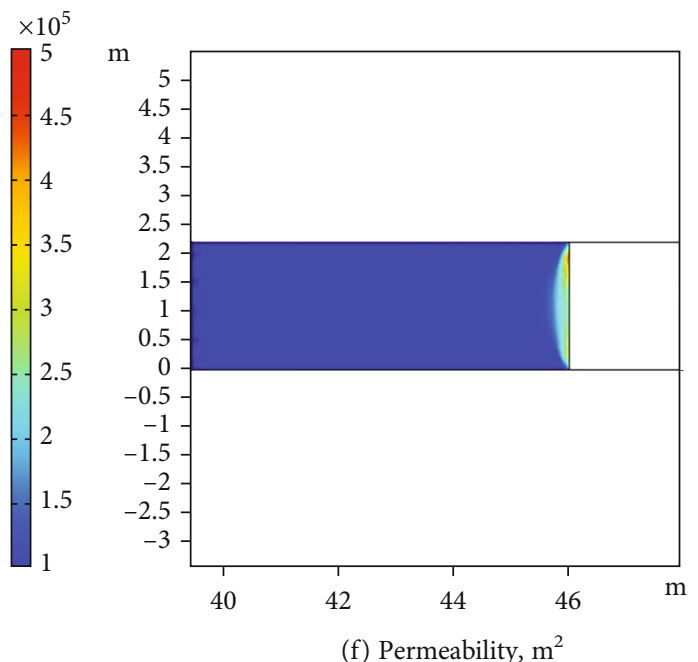

FIgURE 2: Distribution cloud map of physical field in front of working face. 
gas expansion energy in the coal fracture $W_{1}$ and the desorbed gas expansion energy in the coal matrix $W_{2}$

$$
\begin{aligned}
W & =W_{1}+W_{2} \\
& =\frac{p_{0} V_{0 f}}{\kappa-1}\left[\left(\frac{p_{f}}{p_{0}}\right)^{(\kappa-1) / \kappa}-1\right]+\frac{p_{0} V_{0 m}}{\kappa-1}\left[\left(\frac{p_{m}}{p_{0}}\right)^{(\kappa-1) / \kappa}-1\right],
\end{aligned}
$$

where $\kappa$ represents a multiprocess index, usually deemed as 1.25 for this case; $V_{0 f}$ is the gas volume in the fracture, $\mathrm{mL} / \mathrm{g}$; and $V_{0 m}$ is the gas volume desorbed in the outburst in the coal matrix, $\mathrm{mL} / \mathrm{g}$; due to the short outburst time, it generally only lasts for tens of seconds $[14,28]$, that

$$
V_{0 f}=\frac{\phi_{f} p_{f}}{\rho_{c} p_{0}}, V_{0 m}=Q_{s} \frac{V_{M}}{\rho_{c} M_{c}},
$$

where $\rho_{c}$ is the density of coal, $\mathrm{kg} / \mathrm{m}^{3}$. So

$$
\begin{aligned}
W= & \frac{p_{0} V_{f}}{\kappa-1}\left[\left(\frac{p_{f}}{p_{0}}\right)^{(\kappa-1) / \kappa}-1\right] \\
& +\frac{p_{0}}{\kappa-1}\left[\left(\frac{p_{m}}{p_{0}}\right)^{(\kappa-1) / \kappa}-1\right] Q_{s} \frac{V_{M}}{\rho_{c} M_{c}} .
\end{aligned}
$$

3.2. Numerical Calculation Model. The governing equations are imported into COMSOL Multiphysics numerical simulation software for solution and analysis. Solid mechanics module is used to control the deformation of coal and rock, and PDE module is used to analyze the gas flow in coal matrix and fracture. Taking the outburst accident working face of Sanjia coal mine in Guizhou as the background, the buried depth of the working face is $388 \mathrm{~m}$ (the vertical stress is about $10 \mathrm{MPa}$ ), the original gas pressure is $0.86 \mathrm{MPa}$, the thickness of the coal seam on the working face increases from $0.7 \mathrm{~m}$ to $2.2 \mathrm{~m}$, and that is $2.2 \mathrm{~m}$ at the outburst accident site. Therefore, a two-dimensional plane model with $50 \mathrm{~m}$ long, $10 \mathrm{~m}$ high roof and floor, and $2.2 \mathrm{~m}$ thick coal seam is constructed, as shown in Figure 1.

A vertical stress of $10 \mathrm{MPa}$ is applied to the upper part of the geometric model, rollers are used to support the left and right sides, and the bottom is a fixed boundary. The coal seam is excavated $4 \mathrm{~m}$, which is a free boundary. The initial gas pressure of the coal seam is $0.5 \mathrm{MPa}$, the boundary around the coal seam is zero flux, and the impermeable boundary $d p / d x_{i}=0$; after outburst, the outburst hole boundary is atmospheric pressure. The physical parameters of the numerical simulation are shown in Table 1.

\section{Simulation Results}

4.1. Distribution Law of Physical Field in front of Working Face. The vertical stress, volumetric strain, plastic strain, gas pressure, and permeability distribution cloud map of outburst coal face after excavation can be obtained, as shown in Figure 2. The coal and rock mass around the working face

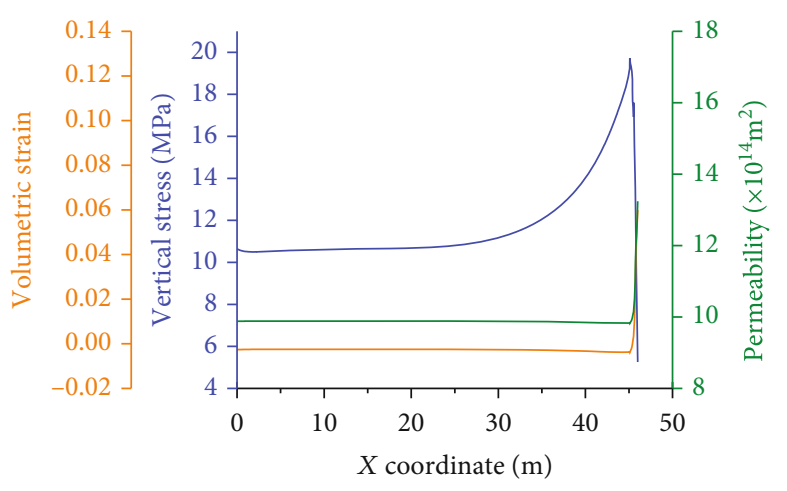

Figure 3: Distribution law of vertical stress, volumetric strain, and permeability in coal seam centerline.

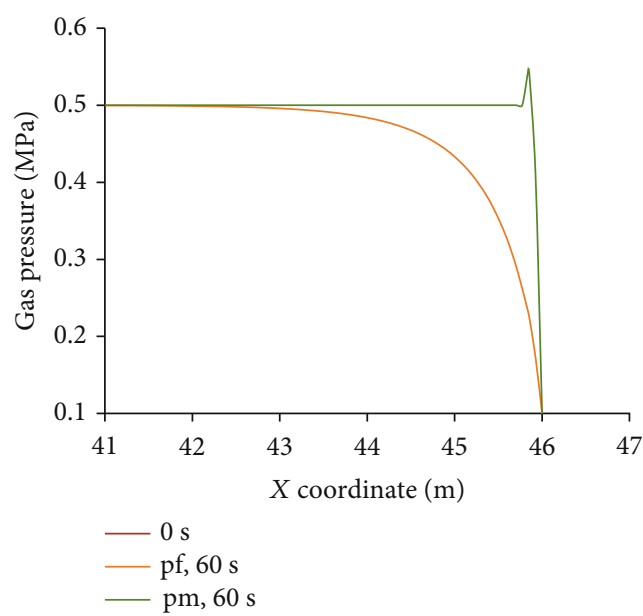

FIGURE 4: Distribution law of gas pressure in coal seam centerline.

suffer from strength failure, plastic deformation, and stress concentration due to high load. Correspondingly, the permeability and gas pressure change. Figure 3 shows the distribution of vertical stress, volumetric strain, and permeability with distance at the coal seam centerline (red line in Figure 1). It can be seen that from the excavation face to the deep part of coal seam, the stress distribution is in turn unloading zone, stress concentration zone, and original stress zone. At the exposed part of the working face, the volume swells deformation is the largest. The coal body is compressed and reaches the minimum at the peak value of vertical stress, then gradually returns to the original stress value along with the stress toward the depth of the coal seam, and the volume strain also gradually returns to 0 . The change of permeability is consistent with the volume strain.

Figure 4 shows the gas pressure distribution of coal matrix and coal fracture at initial time $(0 \mathrm{~s})$ and $60 \mathrm{~s}$. It can be seen from Figure 4 that the gas pressure in coal matrix and coal fracture is the same at the initial time. From the working face to the deep part of the coal seam, the gas pressure rises sharply and reaches the maximum value at the stress peak point, which is slightly higher than the initial pressure of the coal seam. This is because the stress concentration makes the coal seam compacted, which makes it 


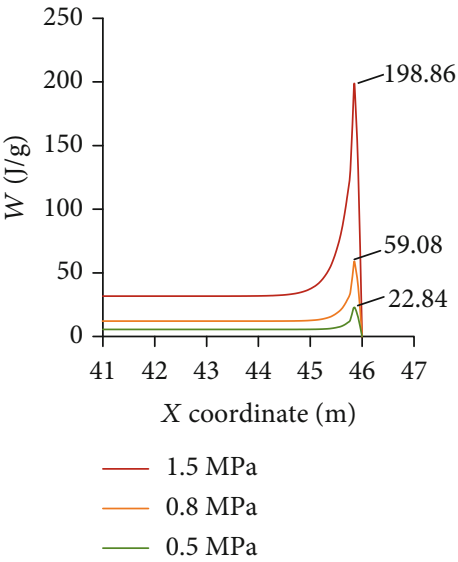

(a) $W$

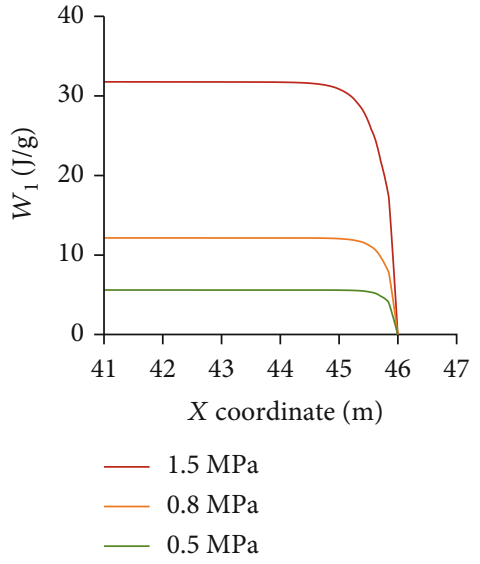

(b) $W_{1}$

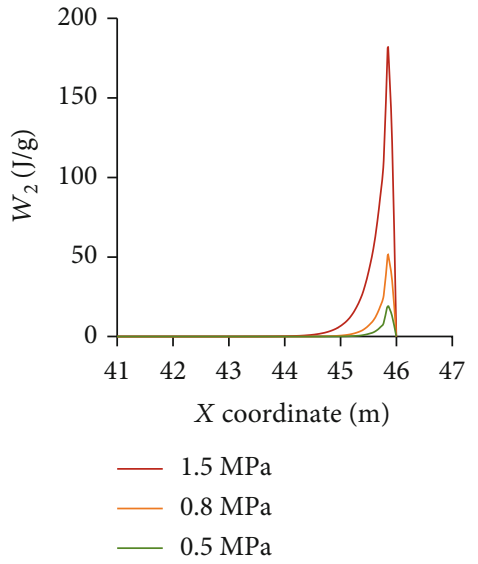

(c) $W_{2}$

FIGURE 5: Expansion energy of gas under different gas pressures.

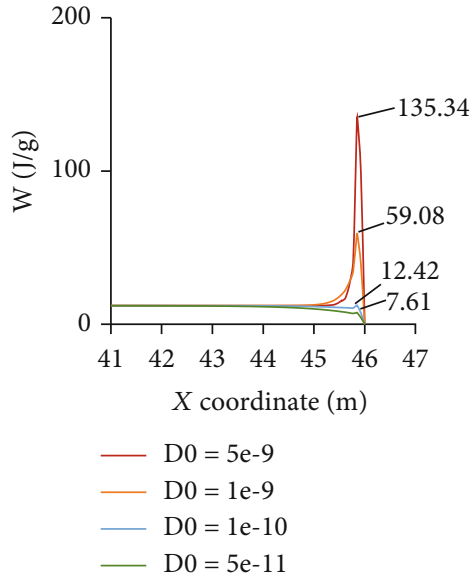

(a) $W$

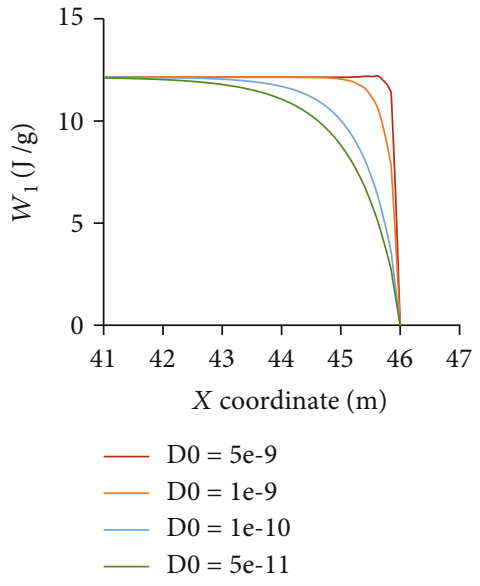

(b) $W_{1}$

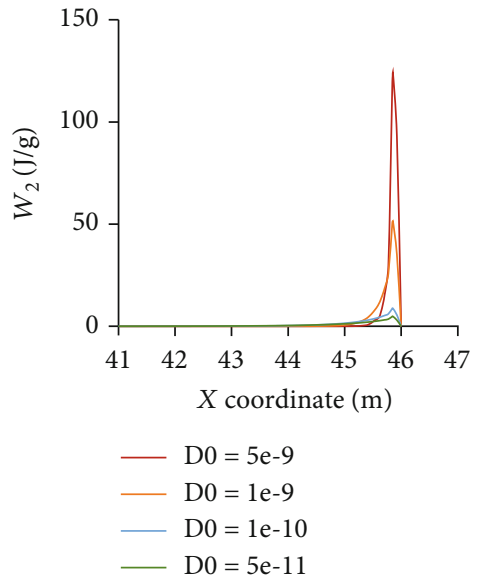

(c) $W_{2}$

FIGURE 6: Expansion energy of gas under different diffusion coefficient.

difficult for the gas to flow outwards and makes the gas accumulate rapidly in a short period of time. Finally, the gas pressure is higher than the initial value in a small range. With the passage of time, the gas in the fracture gradually migrates to the working face under the action of the pressure gradient, and the gas in the coal matrix comes from the gradually desorbs and diffuses of the adsorbed gas of coal particles. When the amount of desorption diffusion in the matrix is less than the gas migration amount in the fracture, the fracture gas pressure begins to decrease, so the coal seam fracture gas pressure near the working face is lower than that in the initial gas pressure at $60 \mathrm{~s}$.

4.2. Influencing Factors of Gas Expansion Energy. The gas expansion energy is directly related to the amount of gas involved in the work. Therefore, it is very important to master the gas flow law under the multifield coupling action of outburst working face. As can be seen from Figure 4, the fracture gas pressure at $5 \mathrm{~m}$ in front of the working face changed greatly, and the outburst duration was generally less than $60 \mathrm{~s}$. Therefore, the gas expansion at $5 \mathrm{~m}$ in front of the work- ing face at the moment of $60 \mathrm{~s}$ was selected for analysis. According to formula (18), the gas expansion energy of outburst coal seam under different conditions can be calculated. The gas expansion energy under different gas pressure is shown in Figure 5. It can be seen that under the simulation conditions, the gas expansion energy of coal seam increases with the increase of gas pressure. Affected by the excavation, the gas expansion energy of the coal seam near the working face is significantly higher than that of the original coal seam. It can be seen from Figure 5(a) that the peak point of gas expansion energy near the working face increases significantly with the increase of gas pressure. The expansion energy provided by free gas keeps a constant value in the original coal seam and decreases gradually near the working face with the distribution of fracture gas pressure. The expansion energy provided by desorption gas is zero in the original coal seam. This is because the coal matrix pores and fractures of the original coal seam have basically the same gas pressure without a concentration gradient, resulting in no new gas is desorbed from the coal matrix. As the increase of gas pressure gradient of coal matrix and fracture near the working 


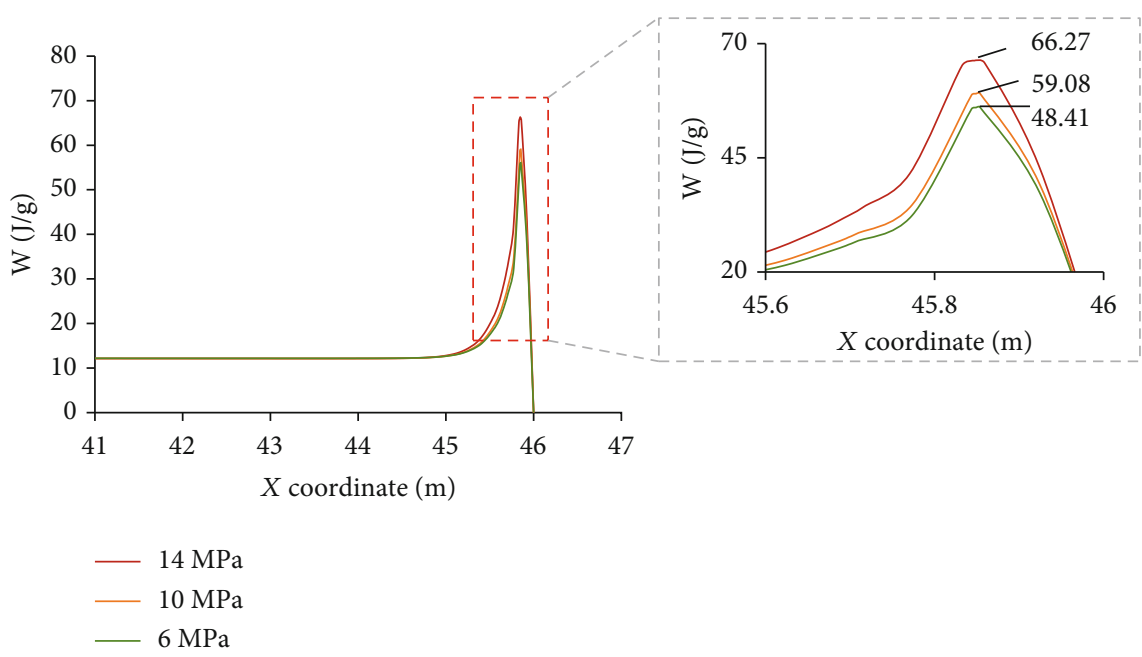

Figure 7: Expansion energy of gas under different stresses.

face, the amount of gas desorbed and diffused increases and decreases to atmospheric pressure at the working face. Therefore, it presents a trend of first increasing and then rapidly decreasing.

Diffusion coefficient is an important parameter of reaction gas diffusion ability, which is related to coal structure, metamorphic degree, particle size, and other factors [29-32]. The gas diffusion ability also has a significant effect on the gas expansion energy, as shown in Figure 6. It can be seen that the gas expansion energy in the original coal seam basically remains unchanged under different diffusion coefficient conditions, about $12.15 \mathrm{~J} / \mathrm{g}$. The peak point of gas expansion energy near the working face increases significantly with the increase of diffusion coefficient. When the diffusion coefficient is less than or equal to $1 \mathrm{e}-10 \mathrm{~m}^{2} / \mathrm{s}$, the decreased value of free gas expansion energy near the working face is greater than that of desorption gas expansion energy, so the gas expansion energy decreases along the $x$-axis. The peak value of gas expansion energy near the working face is $7.61 \mathrm{~J} / \mathrm{g}$, which is less than that of the original coal seam. This is because that when the diffusion coefficient is small, the ability of coal matrix desorption gas diffusion is less than the ability of gas migration to the working face. It can be seen that only when the coal seam has a high diffusion ability, a higher gas expansion energy will be formed near the working face, which has the risk of inducing outburst.

The gas expansion energy under different stress conditions is shown in Figure 7. It can be seen that the stress has little effect on the gas expansion energy under the simulated conditions. The gas expansion energy of original coal seam is not affected by stress. The gas expansion energy near the working face increases with the increase of stress. This may be due to the increase of stress leading to an increase in the degree of coal compaction at the peak stress, which leads to the increase of the gas pressure difference between coal matrix and coal fracture. As a result, the gas desorption diffusion amount of coal matrix increases, so the gas expansion energy increases slightly. The gas expansion energy on the

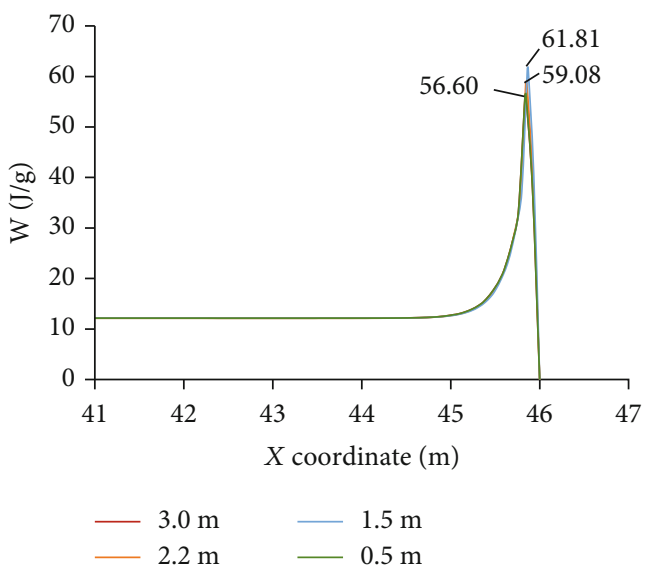

Figure 8: Expansion energy of gas under different thickness of coal seam.

coal seam centerline is not affected by the coal seam thickness, as shown in Figure 8. However, the total gas expansion energy of the whole coal seam is directly related to the thickness, so the possibility of outburst is relatively large when the coal seam thickness changes greatly.

\section{Conclusions}

Considering the outburst coal seam as isotropic elasticplastic porous medium, the multiphysical field gas-solid coupling equation of outburst coal seam was established, which is mainly composed of deformation control equation, gas diffusion control equation in coal matrix, and gas seepage control equation in coal fracture. The numerical analysis model of coal and gas outburst was established by using solid mechanics and PDE custom module of COMSOL Multiphysics, and the distribution law of stress field, deformation field, and gas flow field of coal seam was obtained. Based on these studies, the following conclusions can be drawn: 
(1) From the excavation face to the deep part of coal seam, the stress distribution presents unloading zone, stress concentration zone, and original stress zone. The volumetric strain and permeability reach the minimum at the peak value of vertical stress. The gas pressure in coal matrix and coal fracture is the same at the initial time. Due to the stress concentration, the coal body is compacted, and the gas pressure reaches the maximum at the stress peak point. As time goes on, the gas pressure in the matrix does not change, and the gas pressure in the coal seam fracture near the working face begins to decrease when the gas desorption diffusion amount in the matrix is less than the gas migration amount in the fracture

(2) The total gas expansion energy is affected by the combined effects of free gas and desorption gas expansion energy. The expansion energy provided by free gas is related to the distribution of fracture gas pressure. Affected by the excavation, it maintains a constant value in the original coal seam and gradually decreases in the area close to the working face. The expansion energy provided by desorption gas is zero in the original coal seam. In the vicinity of the working face, it shows a trend of first increases and then decreases rapidly

(3) Compared with stress and coal seam thickness, gas pressure and initial diffusion coefficient have a significant influence on gas expansion energy of coal seam. The expansion energy of coal seam gas increases with the increase of gas pressure. With the increase of diffusion coefficient, the gas expansion energy of the original coal seam is basically unchanged, and the gas expansion energy of the coal seam near the working face increases significantly. When the diffusion coefficient is greater than or equal to $1 \mathrm{e}-9 \mathrm{~m}^{2} / \mathrm{s}$, the gas expansion energy of the coal seam near the working face is significantly higher than that of the original coal seam, which has the risk of inducing outburst

\section{Data Availability}

The data used to support the findings of this study are included within the article.

\section{Conflicts of Interest}

The authors declare that they have no conflicts of interest.

\section{Acknowledgments}

This study was financially supported by the National Natural Science Foundation of China (51974358), the Natural Science Foundation of Chongqing, China (cstc2019jcyjmsxmX0531), the Science and Technology Innovation and Entrepreneurship Fund of China Coal Technology Engineering Group (2019-TD-QN037), the Research Fund of the State Key Laboratory for GeoMechanics and Deep Underground
Engineering, China University of Mining \& Technology (SKLGDUEK1809), and the Science and Technology Project of Chongqing Jiulongpo District (2020-02-008-Y).

\section{References}

[1] В. В. Ходот, Coal and Gas Outburst (Chinese Translation), Beijing: China Industry Press, 1966.

[2] Z. M. Zheng, Mechanism of Coal and Gas Outburst from the Order of Magnitude and Dimension Analysis, Zheng Zhemin's Corpus, Science Press, Beijing, 2004.

[3] G. C. Wen, "Study of coal and gas outburst energy," Mining Safety \& Environmental Protection, vol. 30, no. 6, pp. 1-3, 2003.

[4] H. Lan, J. F. Pan, and Y. W. Peng, "Numerical simulation for energy mechanism of underground dynamic disaster," Journal of China Society, vol. 30, no. S0, pp. 10-14, 2010.

[5] Q. T. Hu and G. C. Wen, The Mechanical Mechanism of Coal and Gas Outburst, Science Press, Beijing, 2013.

[6] G. Wang, M. M. Wu, W. M. Cheng, J. H. Chen, and W. Z. Du, "Analysis of energy conditions for coal and gas outburst and factors influencing outburst intensity," Rock \& Soil Mechanics, vol. 36, no. 10, pp. 2974-2982, 2015.

[7] C. W. Li, B. J. Xie, J. L. Cao, T. T. Wang, and X. Y. Wang, "The energy evalution model of coal and gas outburst intensity," Journal of China Coal Society, vol. 37, no. 9, pp. 1547-1552, 2012.

[8] Q. Tan, S. Yu, H. Zhu, and Z. Zheng, "Fracture of coal containing pressurized gas by sudden relieving," Journal of China Coal Society, vol. 22, pp. 68-72, 1997.

[9] S. Valliappan and W. H. Zhang, "Role of gas energy during coal outbursts," Int J Numer Meth Engng, vol. 44, no. 7, pp. 875-895, 1999.

[10] J. Sobczyk, "The influence of sorption processes on gas stresses leading to the coal and gas outburst in the laboratory conditions," Fuel, vol. 90, no. 3, pp. 1018-1023, 2011.

[11] G. Wang, M. Wu, H. Wang, Q. Huang, and Y. Zhong, "Sensitivity analysis of factors affecting coal and gas outburst based on an energy equilibrium model," Chinese Journal of Rock Mechanics and Engineering, vol. 34, pp. 238-248, 2015.

[12] C. L. Jiang and Q. X. Yu, "Rules of energy dissipation in coal and gas outburst," Journal of China Coal Society, vol. 21, no. 2, pp. 173-178, 1996.

[13] C. Jiang, L. Xu, X. Li et al., "Identification model and indicator of outburst-prone coal seams," Rock Mechanics and Rock Engineering, vol. 48, no. 1, pp. 409-415, 2015.

[14] C. Wang, S. Yang, X. Li, J. Li, and C. Jiang, "Comparison of the initial gas desorption and gas-release energy characteristics from tectonically-deformed and primary-undeformed coal," Fuel, vol. 238, pp. 66-74, 2019.

[15] C. Wang, S. Yang, J. Li, X. Li, and C. Jiang, "Influence of coal moisture on initial gas desorption and gas-release energy characteristics," Fuel, vol. 232, pp. 351-361, 2018.

[16] D. Yang, Y. Chen, J. Tang et al., "Experimental research into the relationship between initial gas release and coal-gas outbursts," Journal of Natural Gas Science and Engineering, vol. 50, pp. 157-165, 2018.

[17] Y. Liang, F. Wang, X. Li, C. Jiang, L. Li, and Y. Chen, "Study on the influence factors of the initial expansion energy of released gas," Process Safety and Environmental Protection, vol. 117, pp. 582-592, 2018. 
[18] S. Xue, C. Zheng, X. Zheng, B. Jiang, Y. Li, and Z. Wang, "Experimental determination of the outburst threshold value of energy strength in coal mines for mining safety," Process Safety and Environmental Protection, vol. 138, pp. 263-268, 2020.

[19] S. Lu, C. Wang, Q. Liu et al., "Numerical assessment of the energy instability of gas outburst of deformed and normal coal combinations during mining," Process Safety and Environmental Protection, vol. 132, pp. 351-366, 2019.

[20] W. Zhao, Y. Cheng, H. Jiang, K. Jin, H. Wang, and L. Wang, "Role of the rapid gas desorption of coal powders in the development stage of outbursts," Journal of Natural Gas Science and Engineering, vol. 28, pp. 491-501, 2016.

[21] T. Xu, C. A. Tang, T. H. Yang, W. C. Zhu, and J. Liu, "Numerical investigation of coal and gas outbursts in underground collieries," International Journal of Rock Mechanics \& Mining Sciences, vol. 43, no. 6, pp. 905-919, 2006.

[22] F.-h. An, Y.-p. Cheng, L. Wang, and W. Li, "A numerical model for outburst including the effect of adsorbed gas on coal deformation and mechanical properties," Computers and Geotechnics, vol. 54, pp. 222-231, 2013.

[23] F. Du and K. Wang, "Unstable failure of gas-bearing coal-rock combination bodies: insights from physical experiments and numerical simulations," Process Safety and Environmental Protection, vol. 129, pp. 264-279, 2019.

[24] Y. Zhao, B. Q. Lin, T. Liu et al., "Mechanism of multifield coupling-induced outburst in mining-disturbed coal seam," Fuel, vol. 272, article 117716, 2020.

[25] Q. Q. Liu, Y. P. Cheng, H. F. Wang et al., "Numerical assessment of the effect of equilibration time on coal permeability evolution characteristics," Fuel, vol. 140, pp. 81-89, 2015.

[26] T. Liu, B. Lin, W. Yang, C. Zhai, and T. Liu, "Coal permeability evolution and gas migration under non-equilibrium state," Transport in Porous Media, vol. 118, no. 3, pp. 393-416, 2017.

[27] X. Cui and R. M. Bustin, "Volumetric strain associated with methane desorption and its impact on coalbed gas production from deep coal seams," AAPG Bulletin, vol. 89, no. 9, pp. 11811202, 2005.

[28] C. L. Zhang, E. Y. Wang, J. Xu, and S. J. Peng, "A new method for coal and gas outburst prediction and prevention based on the fragmentation of ejected coal," Fuel, vol. 287, 2021.

[29] W. Zhao, Y. P. Cheng, Z. J. Pan, K. Wang, and S. M. Liu, "Gas diffusion in coal particles: a review of mathematical models and their applications," Fuel, vol. 252, pp. 77-100, 2019.

[30] Y. Meng and Z. P. Li, "Experimental study on diffusion property of methane gas in coal and its influencing factors," Fuel, vol. 185 , pp. 219-228, 2016.

[31] Q. M. Long, Q. T. Hu, and B. Cheng, "Time-varying diffusion characteristics of different gases in coal particles," International Journal of Mining Science and Technology, vol. 27, no. 6, pp. 1025-1029, 2017.

[32] T. Liu, B. Q. Lin, X. H. Fu et al., "Experimental study on gas diffusion dynamics in fractured coal: a better understanding of gas migration in in-situ coal seam," Energy, vol. 195, p. 117005,2020 . 